\title{
Sum-paintability of generalized theta-graphs
}

\author{
James Carraher* Thomas Mahoney ${ }^{\dagger} \quad$ Gregory J. Puleo ${ }^{\ddagger}$ \\ Douglas B. West ${ }^{\S}$
}

Revised March, 2014

\begin{abstract}
In online list coloring (introduced by Zhu and by Schauz in 2009), on each round the set of vertices having a particular color in their lists is revealed, and the coloring algorithm chooses an independent subset of this set to receive that color. For a graph $G$ and a function $f: V(G) \rightarrow \mathbb{N}$, the graph is $f$-paintable if there is an algorithm to produce a proper coloring when each vertex $v$ is allowed to be presented at most $f(v)$ times.

The sum-paintability of $G$, denoted $\chi_{s p}(G)$, is $\min \left\{\sum f(v): G\right.$ is $f$-paintable $\}$. Basic results include $\chi_{s p}(G) \leq|V(G)|+|E(G)|$ for every graph $G$ and $\chi_{s p}(G)=$ $\left(\sum_{i=1}^{k} \chi_{s p}\left(H_{i}\right)\right)-(k-1)$ when $H_{1}, \ldots, H_{k}$ are the blocks of $G$. Also, adding an ear of length $\ell$ to $G$ adds $2 \ell-1$ to the sum-paintability, when $\ell \geq 3$.

Strengthening a result of Berliner et al., we prove $\chi_{s p}\left(K_{2, r}\right)=2 r+\min \{l+m: l m>$ $r\}$. The generalized theta-graph $\Theta_{\ell_{1}, \ldots, \ell_{k}}$ consists of two vertices joined by internally disjoint paths of lengths $\ell_{1}, \ldots, \ell_{k}$. A book is a graph of the form $\Theta_{1,2, \ldots, 2}$, denoted $B_{r}$ when there are $r$ internally disjoint paths of length 2 . We prove $\chi_{s p}\left(B_{r}\right)=2 r+$ $\min _{l, m \in \mathbb{N}}\left\{l+m: m(l-m)+\left(\begin{array}{c}m \\ 2\end{array}\right)>r\right\}$. We use these results to determine the sumpaintability for all generalized theta-graphs.
\end{abstract}

\section{Introduction}

The list version of graph coloring, introduced by Vizing [12] and Erdös-Rubin-Taylor [3], assigns to each vertex $v$ a set $L(v)$ (called its list) of available colors. An $L$-coloring is a proper coloring $\phi$ such that $\phi(v) \in L(v)$ for each vertex $v$. A graph $G$ is $f$-choosable if $G$ has

\footnotetext{
*Department of Mathematics, University of Nebraska-Lincoln; s-jcarrah1@math.unl.edu.

${ }^{\dagger}$ Department of Mathematics, University of Illinois-Urbana; tmahone2@illinois.edu.

‡Department of Mathematics, University of Illinois-Urbana; gpuleo@gmail.com.

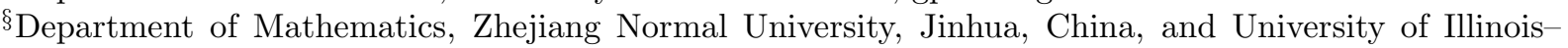
Urbana; west@math.uiuc.edu. Research of D.B. West supported by NSA grant H98230-10-1-0363. Research of J. Carraher supported by NSF grant DMS 09-14815. Research of T. Mahoney and G.J. Puleo supported by by NSF grant DMS 08-38434, "EMSW21-MCTP: Research Experience for Graduate Students".
} 
an $L$-coloring whenever $|L(v)| \geq f(v)$ for each vertex $v$. For $k \in \mathbb{N}$, a graph is $k$-choosable if it is $f$-choosable with $f(v)=k$ for all $v \in V(G)$.

Introduced by Isaak [5], the sum-choosability of a graph $G$, denoted $\chi_{s c}(G)$, is the least $\sum f(v)$ over all $f$ such that $G$ is $f$-choosable. In essence, this studies how large the average list size must be to permit $L$-colorings rather than how large the minimum list size must be. Isaak [5] determined $\chi_{s c}\left(K_{2} \square K_{n}\right)$, and he also observed the easy upper bound $\chi_{s c}(G) \leq$ $|V(G)|+|E(G)|[6]$. Heinold [4] showed that equality does not hold for all outerplanar graphs and also proved results about the sum-choosability of $P_{3} \square P_{n}$.

An online version of list coloring was introduced by Zhu [13]; independently, Schauz [11] introduced an equivalent notion in a game setting. The lists are revealed one color at a time by marking the vertices with that color in their lists. The coloring algorithm, which we call Painter, must choose an independent set of marked vertices to receive that color. Colored vertices will not be marked again; in essence, they are removed from the graph. Each vertex $v$ has a tolerance $f(v)$ for how many times it can be marked.

In the game setting, Painter has an adversary called Lister who specifies the marked sets. Lister wins by marking some vertex $v$ more than $f(v)$ times; Painter wins by producing a proper coloring before this happens. To facilitate discussion, imagine a pile of $f(v)$ tokens at $v$. When Lister marks $v$, one of these tokens is used, and Lister wins if some vertex is marked when it has no tokens. The graph is $f$-paintable if Painter can guarantee winning the game when $f(v)$ tokens are available at each vertex $v$. We often say "Painter can win" to mean "Painter has winning strategy". By analogy with " $k$-choosable" for integer $k$, we say that $G$ is $k$-paintable if $G$ is $f$-paintable when $f(v)=k$ for all $v \in V(G)$, and the paintability or paint number of $G$ is the least $k$ such that $G$ is $k$-paintable.

The behavior of Lister models the need for Painter to win against "worst-case" presentation of lists in online list coloring, so the problems are equivalent. That is, Lister can respond to Painter's choices by not specifying the full lists in advance. Schauz originally called the players "Mr. Paint and Mrs. Correct"; "Marker/Remover" has also been used.

The sum-paintability (or online sum-choosability) of a graph $G$, denoted $\chi_{s p}(G)$, is the least $\sum f(v)$ over all functions $f$ such that $G$ is $f$-paintable. One can imagine Painter being allocated a budget of tokens to distribute to the vertices before the game begins; the sum-paintability is the smallest total that allows Painter to produce a winning distribution.

If $G$ is not $f$-choosable, then $G$ is not $f$-paintable, since Lister can mimic a bad list assignment $L$ by marking in round $i$ the set $\{v \in V(G): i \in L(v)\}$; winning moves by Painter would form an $L$-coloring, which does not exist. Thus, $\chi_{s p}(G) \geq \chi_{s c}(G)$. Nevertheless, $|V(G)|+|E(G)|$ is also an upper bound on sum-paintability (Proposition 2.1).

Letting $\sigma(G)=|V(G)|+|E(G)|$, graphs attaining the upper bound $\sigma(G)$ on sumchoosability or sum-paintability are called sc-greedy or sp-greedy, and $\sigma(G)$ is the greedy bound. Since $\chi_{s c}(G) \leq \chi_{s p}(G) \leq \sigma(G)$, any graph that is sc-greedy is also sp-greedy; however, sp-greediness may be easier to prove. Graphs already shown to be sc-greedy in [1] include cycles, trees (and their line graphs), complete graphs, and graphs whose blocks (maximal 2-connected subgraphs) are all sc-greedy (generalizing [6]).

For strict inequalities, let the generalized theta-graph $\Theta_{\ell_{1}, \ldots, \ell_{k}}$ consist of two vertices joined 
by internally disjoint paths of lengths $\ell_{1}, \ldots, \ell_{k}$. The smallest non-sp-greedy graph is $\Theta_{2,2,2}$, which equals $K_{2,3}$. On the other hand, $\chi_{s c}(G)<\chi_{s p}(G)=\sigma(G)$ for $G=\Theta_{2,2,2 t}$ with $t>1$.

For the analysis of generalized theta-graphs, Erdős, Rubin, and Taylor [3] proved that $\chi_{\ell}\left(\Theta_{2,2,2 t}\right)=2$, so $\chi_{s c}\left(\Theta_{2,2,2 t}\right) \leq 2(2 t+3)=\sigma\left(\Theta_{2,2,2 t}\right)-1$. Heinold [4] showed that equality holds in this bound, but in all other cases, $\chi_{s c}\left(\Theta_{\ell_{1}, \ell_{2}, \ell_{3}}\right)$ is sc-greedy. To study $\chi_{s p}\left(\Theta_{\ell_{1}, \ldots, \ell_{k}}\right.$, we prove a lemma (Lemma 2.8) showing that $\chi_{s p}$ and $\sigma$ increase by the same amount when a path of length at least 3 through new internal vertices of degree 2 is added to a graph. Using this lemma, we determine the sum-paintability of all generalized theta-graphs (Corollary 3.8). (We always index the path lengths so that $\ell_{1} \leq \cdots \leq \ell_{k}$. Since we consider only simple graphs, always $\ell_{2}>1$.)

Berliner et al. [1] proved that $\chi_{s c}\left(K_{2, r}\right)=2 r+\min \{l+m: l m>r\}$. Thus $K_{2, r}$ is "far" from sc-greedy. Strengthening this result, we prove $\chi_{s p}\left(K_{2, r}\right)=\chi_{s c}\left(K_{2, r}\right)$ by showing that Painter can win when $l m>r$, each vertex in the large part has 2 tokens, and the two vertices in the small part have $l$ and $m$ tokens. This follows from a result in [2] that determines who wins in a class of Lister/Painter games of $K_{k, r}$.

The book $B_{r}$ is the graph $\Theta_{\ell_{1}, \ldots, \ell_{r+1}}$ with $\ell_{1}=1$ and $\ell_{2}=\cdots=\ell_{r+1}=2$. We prove $\chi_{s p}\left(B_{r}\right)=2 r+\min \left\{l+m: m(l-m)+\left(\begin{array}{c}m \\ 2\end{array}\right)>r\right\}$. For the lower bound, we prove that $\chi_{s c}\left(B_{r}\right)$ is at least this big. For the upper bound, we give a winning strategy for Painter under a particular allocation of tokens. Using these intermediate results, we determine $\chi_{s p}(G)$ for any generalized theta-graph $G$. While the sum-choosability of generalized theta-graphs is not known, our results provide upper and lower bounds.

\section{Preliminary Results}

Since $f$-paintability implies $f$-choosability, always $\chi_{s p}(G) \geq \chi_{s c}(G)$. Odd cycles have paint number 3 but "just barely" fail to be 2-paintable, in the sense that $\chi_{s p}\left(C_{n}\right)=2 n$ for all $n$ (as noted in the introduction). For ease of describing strategies, we may say that Lister plays $M$ and that Painter deletes an independent subset of $M$.

An easy general upper bound for sum-choosability holds also for sum-paintability.

Proposition 2.1. For any graph $G, \chi_{s p}(G) \leq|V(G)|+|E(G)|$.

Proof. Given any fixed ordering $\pi$ of $V(G)$, Painter allocates $1+d^{-}(v)$ tokens to each vertex $v$, where $d^{-}(v)$ is the number of neighbors of $v$ that occur earlier than $v$ in $\pi$. Painter's strategy is greedy: for any marked set $M$, delete the independent subset of $M$ chosen greedily with respect to $\pi$. That is, the removed set $R$ is the unique maximal set of vertices in $M$ such that each vertex of $M$ is in $R$ if and only if it has no neighbor in $M$ that is earlier in $\pi$.

Painter wins using this strategy, because a vertex $v$ is marked (and not removed) at most once for each earlier neighbor. The total number of tokens is $|V(G)|+|E(G)|$; we have allocated one token for each vertex plus one token for the later endpoint of each edge.

Berliner et al. [1] showed that the sum-choosability of any graph is determined by the sum-choosability of its blocks. 
Theorem 2.2. If $G$ is a graph with blocks $H_{1}, \ldots, H_{k}$, then $\chi_{s c}(G)=\sum_{i=1}^{k} \chi_{s p}\left(H_{i}\right)-(k-1)$.

This statement holds also for sum-paintability. We need a preliminary observation.

Proposition 2.3. Suppose that $G$ is $f$-paintable, where $f$ has sum $\chi_{s p}(G)+t$. Let $\mathcal{S}$ be a winning strategy for Painter under the token assignement $f$. Given that Painter plays according to $\mathcal{S}$, for any vertex $v \in V(G)$ Lister can ensure that at the start of some round, $v$ is still present and has at most $t+1$ tokens.

Proof. If $\mathcal{S}$ allows Painter to guarantee removing $v$ on a round that started with more than $t+1$ tokens on $v$, then playing the same strategy will ensure a win for Painter even when the number of tokens at $v$ is reduced by $1+t$. This gives Painter a winning strategy with $\chi_{s p}(G)-1$ tokens, a contradiction.

Theorem 2.4. If $G$ is a graph with blocks $H_{1}, \ldots, H_{k}$, then $\chi_{s p}(G)=\sum_{i=1}^{k} \chi_{s p}\left(H_{i}\right)-(k-1)$.

Proof. Using induction, it suffices to consider $k=2$. Let $v$ be the one cut-vertex in $G$.

Lower bound: $\chi_{s p}(G) \geq \chi_{s p}\left(H_{1}\right)+\chi_{s p}\left(H_{2}\right)-1$. Let $f$ be an allocation of $\chi_{s p}(G)$ tokens on $G$ that enables Painter to win. Under $f$, let $\ell_{i}$ be the number of tokens on $H_{i}-v$, and let $\ell^{\prime}$ be the number of tokens on $v$.

Since $G$ is $f$-paintable, Painter has a winning strategy when Lister plays only on $H_{i}$. By Proposition 2.3, some strategy for Lister on $H_{1}$ forces the number of tokens on $v$ to be $\ell_{1}+\ell^{\prime}-\chi_{s p}\left(H_{1}\right)+1-q$ for some nonnegative $q$ at the start of some round when $v$ is still present. At that time, $\ell_{1}+\ell^{\prime}-\chi_{s p}\left(H_{1}\right)+\ell_{2}+1-q$ tokens remain on $H_{2}$.

Lister now switches to play on $H_{2}$. By Proposition 2.3, some strategy for Lister on $H_{2}$ forces the number of tokens on $v$ to be at most $\ell_{1}+\ell_{2}+\ell^{\prime}-\chi_{s p}\left(H_{1}\right)-\chi_{s p}\left(H_{2}\right)+2-q$ at the start of some round when $v$ is still present. Since Lister is playing against a winning strategy for Painter and $v$ is still present, this quantity is positive, so $\ell_{1}+\ell_{2}+\ell^{\prime} \geq \chi_{s p}\left(H_{1}\right)+\chi_{s p}\left(H_{2}\right)-1$.

Upper bound: $\chi_{s p}(G) \leq \chi_{s p}\left(H_{1}\right)+\chi_{s p}\left(H_{2}\right)-1$. We give a winning strategy for Painter. For $i \in\{1,2\}$, let $f_{i}$ be a winning allocation of $\chi_{s p}\left(H_{i}\right)$ tokens on $H_{i}$, with $f_{i}(v)=a_{i}$, and let $\mathcal{S}_{i}$ be a strategy that allows Painter to win with this allocation. Define $f$ on $V(G)$ by $f(x)=f_{i}(x)$ for $x \in V\left(H_{i}\right)-\{v\}$ and $f(v)=a_{1}+a_{2}-1$. View $f(v)$ as $\left(a_{1}-1\right)+\left(a_{2}-1\right)+1$, reserving $a_{i}-1$ tokens for $v$ in the game on $H_{i}$, with one left over.

Given Lister's move $M$ on $G$, let $M_{i}=M \cap V\left(H_{i}\right)$, and let $R_{i}$ be the response for Painter dictated by $\mathcal{S}_{i}$. If $v$ is in neither or both of $R_{1}$ and $R_{2}$, then Painter deletes $R_{1} \cup R_{2}$. The game continues under Painter's optimal strategy in both subgraphs independently.

If $v$ lies in exactly one of $R_{1}$ and $R_{2}$, then by symmetry assume $v \in R_{2}$. Painter will not delete $v$. One token has been lost at $v$ since $v \in M$; we view it as a token at $v$ associated with $H_{1}$, and we have $v \in M_{1}$. Painter deletes $R_{1}$ from $H_{1}$; this is the move under $\mathcal{S}_{1}$ in the game on $H_{1}$, including the loss of the token at $v$. In $H_{2}$, Painter pretends that $M_{2}-\{v\}$ was the marked set. Since no token associated with $v$ in $H_{2}$ has been removed from $v$, responding to $M_{2}-\{v\}$ according to $\mathcal{S}_{2}$ continues the winning strategy in $H_{2}$.

By attributing the token removed from $v$ to the appropriate subgame, Painter can use the optimal strategies in the two subgraphs essentially independently. The number of times that 
tokens can be charged to $v$ without removing $v$ is at most $a_{1}-1$ under $\mathcal{S}_{1}$ and at most $a_{2}-1$ under $\mathcal{S}_{2}$. Because these are winning strategies for Painter, $v$ is marked without removal at most $a_{1}+a_{2}-2$ times, and thus a token remains at $v$ at a time when both strategies indicate that $v$ should be removed.

The key to Painter's strategy in Theorem 2.4 is to break Lister's move into subsets associated with the two subgraphs. The subtlety is that how that break is made depends on how Painter's substrategies would respond after the subsets are determined.

Theorem 2.4 allows us to build sp-greedy graphs from smaller sp-greedy graphs, and Theorem 2.2 does the same for sc-greedy graphs.

Corollary 2.5. A graph $G$ is sp-greedy if and only if each of its blocks is sp-greedy, and the same holds for sc-greedy graphs.

In any graph, the subgraph induced by a vertex of degree 1 and its neighbor is a block. Thus adding a pendant edge increases the sum-choosability and the sum-paintability by 2 . This was noted for sum-choosability by Lastrina [9].

Corollary 2.6. If $x$ is a vertex of degree 1 in a graph $G$, then $\chi_{s c}(G)=\chi_{s c}(G-x)+2$ and $\chi_{s p}(G)=\chi_{s p}(G-x)+2$.

Adding an ear to a graph $G$ means adding a path whose endpoints lie in $G$ and whose internal vertices (if any) are new vertices with degree 2. Adding a closed ear is adding a cycle with one vertex in $G$. To study $\chi_{s p}$ under addition of ears, we need an observation about degeneracy that is used in [2] and in earlier papers on paintability such as $[7,8,13]$. It states that vertices with "excess" tokens are irrelevant in determining whether a graph is $f$-paintable.

Proposition 2.7. Let $v$ be a vertex in a graph $G$ with token assignment $f$. Let $G^{\prime}=G-v$, and let $f^{\prime}$ be the restriction of $f$ to $V\left(G^{\prime}\right)$. If $f(v)>d_{G}(v)$, then $G$ is $f$-paintable if and only if $G^{\prime}$ is $f^{\prime}$-paintable.

Lemma 2.8. If $G^{\prime}$ is obtained from $G$ by adding an ear or closed ear with $m$ edges, where $m \geq 3$, then $\chi_{s p}\left(G^{\prime}\right)=\chi_{s p}(G)+2 m-1$.

Proof. We first reduce to the case $m=3$. If the ear is longer, then we first grow a path of length $m-3$ from one endpoint of the ear in $G$. By Corollary 2.6, this adds $2(m-3)$ to the sum-paintability. If the claim holds when $m=3$, then adding the last three edges requires five more tokens, yielding $\chi_{s p}\left(G^{\prime}\right)=\chi_{s p}(G)+2 m-1$.

Hence we may assume $m=3$. Let $x$ and $y$ be the internal vertices of the ear. If $G$ is $f$-choosable, then extend $f$ to $G^{\prime}$ by letting $f(x)=2$ and $f(y)=3$. Since $f(y)>d_{G^{\prime}}(y)$ and $f(x)>d_{G^{\prime}-y}(x)$, by Proposition $2.7 G^{\prime}$ is $f$-paintable. Hence $\chi_{s p}\left(G^{\prime}\right) \leq \chi_{s p}(G)+5$.

Let $f$ be an allocation of fewer than $\chi_{s p}(G)+5$ tokens to $G^{\prime}$. We prove that Lister can win by playing the new vertices or can use them to reduce the number of tokens on $G$ below $\chi_{s p}(G)$ before starting to play there. By symmetry, we may assume that $f(x) \leq f(y)$. Let $x^{\prime}$ and $y^{\prime}$ be the neighbors in $V(G)$ of $x$ and $y$, respectively $\left(x^{\prime}=y^{\prime}\right.$ if the ear is closed). 
If $f(x)+f(y) \geq 5$, then Lister can win by playing only on $G$. If $f(x)=0$, then Lister plays $\{x\}$ and immediately wins. If $f(x)=f(y)=1$, then Lister plays $\{x, y\}$ to win. If $f(x)=1$ and $f(y)=2$, then Lister plays $\left\{x^{\prime}, x, y\right\}$, forcing removal of $x$. Now $y$ has one token remaining; Lister plays $\left\{y, y^{\prime}\right\}$, forcing removal of $y$. Now only $G$ remains, with fewer than $\chi_{s p}(G)$ tokens, and Lister can win under any such distribution on $G$.

Finally, when $f(x)+f(y)=4$, it suffices for Lister to reduce the token count on $G$ by 1 without Painter deleting any vertices of $G$. If $f(x)=1$, then Lister plays $\left\{x, x^{\prime}\right\}$. If $f(x)=f(y)=2$, then Lister first plays $\{x, y\}$, forcing removal of one of them; by symmetry, let it be $x$. Now $y$ has one remaining token; Lister plays $\left\{y, y^{\prime}\right\}$, forcing removal of $y$. Now only $G$ remains, with fewer than $\chi_{s p}(G)$ tokens, and Lister can win the remaining game.

We never ask Lister to play both $x^{\prime}$ and $y^{\prime}$, so it does not matter whether the ear is closed. In all cases, Lister can win if $\sum_{v \in V(G)} f(v)<\chi_{s p}(G)+5$, so $\chi_{s p}\left(G^{\prime}\right) \geq \chi_{s p}(G)+5$.

The conclusion of Lemma 2.8 does not hold for ears of length at most 2. For example, $\Theta_{2,2}$ is $C_{4}$, which is sp-greedy, but the graph $\Theta_{2,2,2}$ obtained by adding one ear of length 2 is not sp-greedy, as shown by Theorem 3.2.

Using Lemma 2.8 and Corollary 2.6, one can generate many sp-greedy graphs. It is well known that every 2-edge-connected graph $G$ can be grown from any cycle in $G$ by iteratively adding ears or closed ears.

Corollary 2.9. If $G$ is a 2-edge-connected graph having an ear decomposition in which every ear or closed ear has length at least 3 , then $G$ is sp-greedy. If $G$ arises from a subgraph $H$ such that $\chi_{s p}(H)=\sigma(H)-t$ by such additions, then $\chi_{s p}(G)=\sigma(G)-t$.

Corollary 2.10. For $t>1$, the graph $\Theta_{2,2,2 t}$ is sp-greedy but not sc-greedy.

Proof. For $G=\Theta_{2,2,2 t}$, Heinold [4] proved $\chi_{s c}(G)=\sigma(G)-1$. Applying Lemma 2.8 to the ear of length $2 t$ yields $\chi_{s p}(G)=\chi_{s p}\left(C_{4}\right)+4 t-1=4 t+7=\sigma(G)$, since $C_{4}$ is sp-greedy.

Corollary 2.10 provides another proof of the result in [13] that $\Theta_{2,2,2 t}$ is not 2-paintable for $t>1$. As we have noted, Heinold [4] showed that all other graphs of the form $\Theta_{\ell_{1}, \ell_{2}, \ell_{3}}$ are sc-greedy and thus also sp-greedy.

\section{Main results}

Toward the sum-paintability of the generalized theta-graph, note first that $\Theta_{k_{1}, \ldots, k_{r}}=K_{2, r}$ when $k_{1}=\cdots=k_{r}=2$. From [1], we have $\chi_{s c}\left(K_{2, r}\right)=2 r+\min \{l+m: l m>r$ and $l, m \in$ $\mathbb{N}\}$. We show that $\chi_{s p}\left(K_{2, r}\right)=\chi_{s c}\left(K_{2, r}\right)$ by using a characterization of Painter winnability for a special family of Lister-Painter games on complete bipartite graphs.

Theorem 3.1 ([2]). Consider $K_{k, r}$ with $k \leq r$. Let $X$ and $Y$ be the partite sets, with $X=x_{1}, \ldots, x_{k}$. If $f\left(x_{i}\right)=t_{i}$ for $1 \leq i \leq k$, and $f(w)=k$ for $w \in Y$, then Painter has a winning strategy if and only if $r<\prod_{i=1}^{k} t_{i}$.

Theorem 3.2. If $r \geq 2$, then $\chi_{s p}\left(K_{2, r}\right)=2 r+\min \{l+m: l m>r$ and $l, m \in \mathbb{N}\}$. 
Proof. Since always $\chi_{s p}(G) \geq \chi_{s c}(G)$, it suffices to find an allocation of $2 r+l+m$ tokens (whenever $l m>r$ ) that enables Painter to win. Allocate two tokens to each vertex in the partite set of size $r$. Allocate $l$ and $m$ tokens, respectively, to the two vertices in the other partite set. By Theorem 3.1, Painter has a winning strategy if and only if $l m>r$.

Since the product of two values with fixed sum is maximized when they are equal, we expect $\min \{l+m\}$ in the formula to be about $2 \sqrt{r}$. More precisely, Heinold [4] showed that the value in Theorem 3.2 always equals $2 r+1+\lfloor\sqrt{4 r+1}\rfloor$.

To prove $\chi_{s p}(G)>k$, we must show that $G$ is not $f$-paintable for any token assignment $f$ with sum $k$. Like Proposition 2.7, the next lemma reduces the number of token assignments that need to be considered. It is the paintability analogue of an observation made by Heinold [4] that we subsequently state and use.

Lemma 3.3. Let $v$ be a vertex in a graph $G$. For $f: V(G) \rightarrow \mathbb{N}$ such that $f(v)=1$, the graph $G$ is $f$-paintable if and only if $G-v$ is $f^{\prime}$-paintable, where $f^{\prime}(u)=f(u)$ for $u \notin N(v)$ and $f^{\prime}(u)=f(u)-1$ for $u \in N(v)$.

Proof. Lister may begin by marking $\{v\} \cup N(v)$. Because Painter then must delete $v$, Painter can guarantee winning the game only if $G-v$ is $f^{\prime}$-paintable.

Conversely, suppose that $G-v$ is $f^{\prime}$-paintable. In the game on $G$, Painter must delete $v$ when it is first marked. The extra token given to each neighbor of $v$ is reserved for the round on which $v$ is first marked and removed. By playing the winning strategy for $G-v$ under $f^{\prime}$ in the other rounds, Painter guarantees winning on $G$ under $f$.

Lemma 3.4 ([4]). The statement of Lemma 3.3 also holds when "paintable" is replaced by "choosable".

Recall that the book $B_{r}$ is the graph $\Theta_{\ell_{1}, \ldots, \ell_{r+1}}$ with $\ell_{1}=1$ and $\ell_{2}=\cdots=\ell_{r+1}=2$. We obtain the lower bound on $\chi_{s p}\left(B_{r}\right)$ by proving a lower bound on $\chi_{s c}\left(B_{r}\right)$. We will see in Proposition 3.7 that the bound is a bit more than $2 r+\sqrt{6 r}$. Let $[n]=\{1, \ldots, n\}$.

Lemma 3.5. $\chi_{s c}\left(B_{r}\right) \geq g(r)$, where $g(r)=2 r+\min \left\{l+m: m(l-m)+\left(\begin{array}{c}m \\ 2\end{array}\right)>r\right\}$.

Proof. We use induction on $r$. If $r=1$, then $B_{r}=K_{3}$ and $\chi_{s c}\left(K_{3}\right)=6=g(1)$. For $r>1$, we show that $f$-choosability of $B_{r}$ requires $\sum f(v) \geq g(r)$. Let $t=\sum_{v \in V(G)} f(v)$.

Let $x$ and $y$ be the two dominating vertices in $B_{r}$, and let $S$ be the set of $r$ vertices of degree 2. First suppose that $f(v)=2$ for all $v \in S$, with $f(x)=l$ and $f(y)=m$, where $l \geq m$. Note that $t=2 r+l+m$. If $t<g(r)$, then $m(l-m)+\left(\begin{array}{c}m \\ 2\end{array}\right) \leq r$. Let $L(x)=[l]$ and $L(y)=[m]$. Assign distinct lists from $\left(\begin{array}{c}{[m]} \\ 2\end{array}\right)$ to the first $\left(\begin{array}{c}m \\ 2\end{array}\right)$ vertices of $S$, distinct lists from $[m] \times([l]-[m])$ to the next $m(l-m)$ vertices of $S$, and list $\{1,2\}$ to any remaining vertices of $S$. In an $L$-coloring, the colors on $x$ and $y$ (which must be distinct) form the list for some vertex of $S$. Hence an $L$-coloring cannot be completed, and $B_{r}$ is not $f$-choosable.

We may now assume that $f(v) \neq 2$ for some $v \in S$. First suppose that $f(v)=1$ and $B_{r}$ is $f$-choosable. Note that $B_{r}-v=B_{r-1}$. By Lemma 3.4, $B_{r}$ is $f$-choosable only if $B_{r-1}$ is $f^{\prime}$-choosable, where $f^{\prime}(u)=f(u)-1$ for $u \in\{x, y\}$ and otherwise $f^{\prime}(u)=f(u)$. By the induction hypothesis, this requires $\sum f^{\prime}(u) \geq g(r-1)$. Hence $t \geq 3+g(r-1) \geq g(r)$. 
If $f(v) \geq 3$ and $B_{r}$ is $f$-choosable, then $B_{r}-v$ is $f^{\prime}$-choosable, where $f^{\prime}$ is the restriction of $f$ to $V\left(B_{r}\right)-\{v\}$. Hence again $t \geq 3+g(r-1) \geq g(r)$.

For the upper bound on $\chi_{s p}\left(B_{r}\right)$, we give a winning strategy for Painter under a token assignment with sum $g(r)$.

Theorem 3.6. $\chi_{s p}\left(B_{r}\right)=g(r)$, where $g(r)=2 r+\min \left\{l+m: m(l-m)+\left(\begin{array}{c}m \\ 2\end{array}\right)>r\right\}$.

Proof. Theorem 3.5 gives the lower bound. For the upper bound, allocate $g(r)$ tokens by setting $f(v)=2$ for $v \in S$ and letting $f(x)=l$ and $f(y)=m$, where $l$ and $m$ are any integers such that $m(l-m)+\left(\begin{array}{c}m \\ 2\end{array}\right)>r$; call this inequality $(*)$ for $B_{r}$. For fixed $\{l, m\}$, the left side of $(*)$ is largest when $l \geq m$; since $l+m$ is symmetric, Painter may assume $l \geq m$. We prove by induction on $r$ that Painter has a winning strategy under any such allocation.

When $r=1$, Painter has a winning strategy (on $K_{3}$ ) when $l \geq 3$ and $m \geq 1$. Now consider $r>1$. Let $M$ be the marked set presented by Lister in the first round. We consider possible choices for $M$. Let $S=V\left(B_{r}\right)-\{x, y\}$ and $k=|S \cap M|$.

Case 1: $x, y \notin M$. The marked set $M$ is independent; Painter deletes $M$. By the induction hypothesis, Painter can win in the remaining graph $B_{r-k}$.

Case 2: $|M \cap\{x, y\}|=1$. Let $\left\{z, z^{\prime}\right\}=\{x, y\}$, with $z$ being the marked vertex in $\{x, y\}$. If $k<f\left(z^{\prime}\right)$, then Painter deletes $z$, leaving a star. By Proposition 2.7, the vertices of $S-M$ are now irrelevant. The remaining graph is a star having $k$ leaves with one token at each and center $z^{\prime}$ having $f\left(z^{\prime}\right)$ tokens; since $f\left(z^{\prime}\right)>k$, Painter has a winning strategy.

If $k \geq f\left(z^{\prime}\right)$, then Painter deletes $S \cap M$, which leaves the graph $B_{r-k}$ with $f(z)-1$ tokens on $z$ and $f\left(z^{\prime}\right)$ tokens on $z^{\prime}$ (and two tokens each on the remaining vertices). The right side of $(*)$ decreases by $k$. The left side of $(*)$ decreases by at most $k$ (by $m$ if $z=x$ and by $l-m$ if $z=y)$. Hence $(*)$ is satisfied for $B_{r-k}$, and the induction hypothesis applies.

Case 3: $x, y \in M$. Recall that $l \geq m$. If $k<l-1$, then Painter deletes $y$, leaving a star. As in Case 2, we reduce to a star having $k$ leaves with one token at each and center with more than $k$ tokens; Painter has a winning strategy.

If $k \geq l-1$, then Painter deletes $S \cap M$, which leaves the graph $B_{r-k}$ with $l-1$ and $m-1$ tokens on $x$ and $y$ (and two tokens each on the remaining vertices). The right side of (*) decreases by $k$ and the left side by $l-1$, which is at most $k$. Hence $(*)$ is satisfied for $B_{r-k}$, and the induction hypothesis applies.

Proposition 3.7. Always $h_{r} \leq g(r) \leq h_{r}+1$, where $h_{r}=\left\lceil 2 r+\frac{1}{2}+\sqrt{6 r}\right\rceil$.

Proof. To perform the optimization in the value of $g(r)$, first consider the continuous relaxation. Let $h=l+m$. Fixing $h$, the required condition for $l$ and $m$ simplifies to $m(2 h-1-3 m)>2 r$. Choosing $m$ to maximize the left side, we find that $h>\frac{1}{2}+\sqrt{6 r}$ is necessary. This requirement applies also to the integer restriction, so $g(r) \geq h_{r}$.

If $m=\sqrt{2 r / 3}$ and $l=\frac{1}{2}+2 \sqrt{2 r / 3}$, then equality holds: $m(l-m)+\left(\begin{array}{c}m \\ 2\end{array}\right)=r$. In this case $l+m=\frac{1}{2}+\sqrt{6 r}$. However, integer values are needed, and these values cannot both be integers. In all cases, setting $m^{\prime}=\lceil\sqrt{2 r / 3}\rceil$ and $l^{\prime}=\left\lceil\frac{1}{2}+2 \sqrt{2 r / 3}\right\rceil$ yields $m^{\prime}\left(l^{\prime}-m^{\prime}\right)+$ $\left(\begin{array}{c}m^{\prime} \\ 2\end{array}\right)>r$ and $l^{\prime}+m^{\prime} \leq 2+\sqrt{6 r}$. In an interval of length $3 / 2$, there are at most two integers, in this case $h_{r}$ and $h_{r}+1$. 
Computation for $r \leq 1,000,000$ shows that each of the values $h_{r}$ and $h_{r}+1$ is correct about half of the time.

Corollary 3.8. If $G$ is a generalized theta-graph $\Theta_{\ell_{1}, \ldots, \ell_{r}}$, then

$$
\chi_{s p}(G)= \begin{cases}\sigma(G), & \text { if } t \leq 3 \\ \chi_{s p}\left(K_{2, t-1}\right)+\sum_{i=t}^{r}\left(2 \ell_{i}-1\right), & \text { if } \ell_{1}=2 \text { and } t>3 \\ \chi_{s p}\left(B_{t-2}\right)+\sum_{i=t}^{r}\left(2 \ell_{i}-1\right), & \text { if } \ell_{1}=1 \text { and } t>3\end{cases}
$$

where $t=r+1$ when $\ell_{r} \leq 2$ and otherwise $t=\min \left\{i: \ell_{i}>2\right\}$. In particular, a generalized theta-graph $G$ is sp-greedy if and only if $t \leq 3$ or if $\ell_{1}=1$ and $t \leq 5$.

Proof. First we calculate the sum-paintability of the subgraph formed by two shortest paths (when $\ell_{3}>2$ ) or by all paths of length at most 2 (when $\ell_{3}=2$ ), using $\chi_{s p}\left(C_{n}\right)=2 n$, Theorem 3.2, or Theorem 3.6. We then apply Lemma 2.8 to add the remaining paths. Each remaining path of length $\ell_{i}$ contributes $2 \ell_{i}-1$ to the sum-paintability.

If $t \leq 3$, then we have argued that $G$ is sp-greedy. If $t \in\{4,5\}$ and $\ell_{1}=1$, then again $G$ is sp-greedy, because $B_{t-2}$ is sp-greedy. If $t \geq 4$ and $\ell_{1}=2$, then $G$ is not sp-greedy, because $\chi_{s p}\left(K_{2, t-1}\right)<\sigma\left(K_{2, t-1}\right)$ (by Theorem 3.2). If $t>5$ and $\ell_{1}=1$, then $G$ is not sp-greedy, because $\chi_{s p}\left(B_{t-2}\right)<\sigma\left(B_{t-2}\right)$ (by Theorem 3.6).

Although $\chi_{s c}\left(K_{2, r}\right)=\chi_{s p}\left(K_{2, r}\right)$ and $\chi_{s c}\left(B_{r}\right)=\chi_{s p}\left(B_{r}\right)$, the sum-choosability and sumpaintability of generalized theta-graphs need not be equal. We noted earlier that $\chi_{s c}\left(\Theta_{2,2,2 t}\right)<$ $\chi_{s p}\left(\Theta_{2,2,2 t}\right)=\sigma\left(\Theta_{2,2,2 t}\right)$ for $t>1$. The reason is that Lemma 2.8 does not hold for sumchoosability. To obtain a lower bound on $\chi_{s c}\left(\Theta_{\ell_{1}, \ldots, \ell_{r}}\right)$, we instead start with $K_{2, r}, B_{r}$, or a cycle (where the sum-choosability is known) and apply Corollary 2.6 to add pendant paths (ears with a missing edge). Each path of length $\ell_{i}$ increases the sum-choosability by at least $2\left(\ell_{i}-1\right)$. We do not know when adding the last edge of an ear increases the sum-choosability. This argument yields the following corollary.

Corollary 3.9. If $G$ is a generalized theta-graph $\Theta_{\ell_{1}, \ldots, \ell_{r}}$, then $\chi_{s c}(G) \geq \chi_{s p}(G)-r+t-1$, where $t$ is 3 when $\ell_{2}>2$, is $r+1$ when $\ell_{r} \leq 2$, and otherwise is $\min \left\{i: \ell_{i}>2\right\}$.

\section{References}

[1] A. Berliner, U. Bostelmann, R. A. Brualdi, and L. Deaett, Sum list coloring graphs. Graphs and Comb. 22 (2006), 173-183.

[2] J. Carraher, S. Loeb, T. Mahoney, G. Puleo, M. Tsai, D. West. Three topics in online list coloring, submitted.

[3] P. Erdős, A.L. Rubin, and H. Taylor, Choosability in graphs. Proc. West Coast Conf. Combinatorics, Graph Theory and Computing (Humboldt State Univ., Arcata, Calif., 1979), Congr. Numer. 26 (1980) 125-157. 
[4] B. Heinold, Sum List Coloring and Choosability. PhD Thesis, Lehigh University, 2006.

[5] G. Isaak, Sum list coloring $2 \times n$ arrays. Electron. J.Combin. 9 (2002), \#N8.

[6] G. Isaak, Sum list coloring block graphs. Graphs Combin. 20 (2004), 499-506.

[7] S.-J. Kim, Y. Kwon, D.D. Liu, and X. Zhu, On-line list colouring of complete multipartite graphs. Electron. J.Combin. 19 (2012), Paper \#P41, 13 pages.

[8] J. Kozik, P. Micek, and X. Zhu, Towards On-line Ohba's Conjecture, preprint.

[9] M. Lastrina, List-coloring and sum-list-coloring problems on graphs. Graduate Theses and Dissertations (2012), Paper 12376.

[10] T. Mahoney, C. Tomlinson, and J.I. Wise, Families of online sum-choice-greedy graphs, in preparation.

[11] U. Schauz, Mr. Paint and Mrs. Correct. Electron. J.Combin. 16 (2009) \#R77.

[12] V. G. Vizing, Vertex colorings with given colors (Russian). Diskret. Analiz. 29 (1976), $3-10$.

[13] X. Zhu, On-Line List Colouring of Graphs. Electron. J. Combin. 16(1) (2009) \#R127. 